\title{
AWMF fordert in einer Resolution die Anerkennung der Leitlinienarbeit als wissenschaftliche Forschungsleistung
}

\section{Zusammenfassung}

Mit großer Mehrheit hat die Delegiertenkonferenz der Arbeitsgemeinschaft der Wissenschaftlichen Medizinischen Fachgesellschaften (AWMF) am 9. November 2013 in Frankfurt/Main eine Resolution zur Anerkennung der Leitlinienarbeit als originäre wissenschaftliche Forschungsleistungen verabschiedet. Die Resolution wendet sich an alle medizinischen Fakultäten, andere Forschungseinrichtungen und Organisationen der Forschungsförderung und fordert dazu auf, Wissenschaftlerinnen und Wissenschaftler nicht zu benachteiligen, die sich an der wichtigen wissenschaftlichen Arbeit der Leitlinienerstellung beteiligen.

\author{
Wolfgang Müller ${ }^{1}$
}

1 AWMF-Geschäftsstelle

\section{Text}

Die Delegierten der über 160 Mitgliedsgesellschaften der AWMF billigten mit großer Mehrheit folgenden Resolutionstext:

"Die Delegiertenkonferenz der Arbeitsgemeinschaft der Wissenschaftlichen Medizinischen Fachgesellschaften (AWMF) fordert die Anerkennung der Erstellung und Aktualisierung von Leitlinien und Systematischen Übersichtsarbeiten, die nach wissenschaftlichen Kriterien erarbeitet wurden, als originäre wissenschaftliche Forschungsleistungen."

\section{Zur Begründung der Resolution heißt es:}

"International werden systematisch entwickelte Leitlinien (Clinical Practice Guidelines) und systematische Übersichtsarbeiten (Systematic Reviews) als wesentliche Instrumente des Wissensmanagements und der Qualitätsförderung im Gesundheitswesen angesehen. Dies schlägt sich auch in der Schaffung wissenschaftlicher Strukturen zu ihrer Förderung nieder. Daraus leitet sich die Forderung nach mehr Meta-Forschung in der Medizin ab, deren Aufgabe durch systematische Recherchen existierenden Wissens, dessen kritische Analyse, kontextuelle Einordnung sowie die konzeptionelle Implementierung relevanter neuer Erkenntnisse und Konkretisierung weiteren Forschungsbedarfs charakerisiert ist. Diese ist nicht nur für die Patientenversorgung relevant sondern trägt auch zur Weiterentwicklung der medizinischen Forschung bei. Leitlinien sind die wichtigsten effektiven Instrumente für den Transfer von Ergebnissen der patientennahen klinischen Forschung in die Gesundheitsversorgung.

Diese Sichtweise in der Beurteilung der Forschungsleistungen von Autoren von Leitlinien und systematischen Übersichtsarbeiten (wie zum Beispiel Cochrane Reviews, Health Technology-Assessment Reports) hat sich in Deutschland immer noch nicht durchgesetzt.
Ohne die interdisziplinäre, breit vernetzte und vielfach ehrenamtliche Kooperation von Wissenschaftlern und klinisch Tätigen lassen sich Leitlinien und Systematische Reviews zum Nutzen der Patienten nicht entwickeln. Daher ist es für die AWMF nicht akzeptabel, dass daran mitwirkende Wissenschaftler bisweilen sogar Nachteile für ihre individuelle Karriere erleiden.

Die wissenschaftliche Anerkennung entsprechend dem internationalen Niveau wird die internationale Vergleichbarkeit von Forschungsleistungen erleichtern, sowohl bei der Beurteilung individueller Wissenschaftler als auch bei der Beurteilung von wissenschaftlichen Einrichtungen.

Medizinische Fakultäten und andere Forschungseinrichtungen und -förderer sind daher aufgefordert, die Arbeit an Leitlinien und Systematischen Übersichtsarbeiten als wissenschaftliche Forschungsleistung anzuerkennen. Zur Bewertung von Publikationsleistungen von Leitlinien und Systematischen Reviews hat die Kommission „Leistungsevaluation von Forschung und Lehre“ der AWMF Empfehlungen bereit gestellt."

\section{Weitere Informationen:}

Empfehlungen der AWMF-Kommission "Leistungsevaluation von Forschung und Lehre":

www.awmf.org/forschung-lehre/komm-evaluation-von-fl/ leistungsevaluation-forschung/bibliometrie/ reviews-metaanalysen-leitlinien.html

\section{Korrespondenzadresse:}

M.A. Wolfgang Müller

AWMF-Geschäftsstelle, Ubierstr. 20, 40223 Düsseldorf office@awmf.org 
Bitte zitieren als

Müller W. AWMF fordert in einer Resolution die Anerkennung der Leitlinienarbeit als wissenschaftliche Forschungsleistung. GMS Mitt AWMF. 2013;10:Doc15.

DOI: 10.3205/awmf000288, URN: urn:nbn:de:0183-awmf0002883

Artikel online frei zugänglich unter

http://www.egms.de/en/journals/awmf/2013-10/awmf000288.shtml
Eingereicht: 11.11.2013

\section{Copyright}

(C2013 Müller. Dieser Artikel ist ein Open Access-Artikel und steht unter den Creative Commons Lizenzbedingungen

(http://creativecommons.org/licenses/by-nc-nd/3.0/deed.de). Er darf vervielfältigt, verbreitet und öffentlich zugänglich gemacht werden, vorausgesetzt dass Autor und Quelle genannt werden. 